\title{
Effect of Add-On Therapy of Dapagliflozin and Empagliflozin on Adipokines in Type 2 Diabetes Mellitus
}

\author{
Abid Shaheer ${ }^{\mathrm{a}, \mathrm{e}} \mathbb{1}$, Ashok Kumar ${ }^{\mathrm{b}}$, Palat Menon ${ }^{\mathrm{c}}$, Mahir Jallo ${ }^{\mathrm{d}}$, Shaikh Basha ${ }^{\mathrm{d}}$
}

\begin{abstract}
Background: The alteration of adipokine secretion leads to the development of insulin resistance or impaired function of insulin in type 2 diabetes with obesity. The main objective of the study was to evaluate the effect of add-on therapy of dapagliflozin and empagliflozin on visceral fat-associated adipokines in inadequately controlled overweight and obese type 2 diabetic patients on metformin monotherapy.
\end{abstract}

Methods: The study included 60 participants diagnosed with type 2 diabetes mellitus with overweight or obesity. The blood samples were taken before starting first-line therapy with metformin, 12 weeks after starting metformin therapy and 12 weeks after starting add-on therapy. The biochemical variables were analyzed using Cobas ${ }^{\circledR} 6000$ analyzer. Hemoglobin A1c (HbA1c) level was measured with highperformance liquid chromatography (HPLC). Serum adipokines were estimated with enzyme-linked immunosorbent assay (ELISA).

Results: The mean adiponectin level was significantly elevated with add-on therapy using dapagliflozin and empagliflozin $(\mathrm{P}<0.001)$. The mean fatty-acid binding protein 4 (FABP4), retinol-binding protein 4 (RBP4) and visfatin levels were reduced considerably ( $\mathrm{P}$ $<0.001)$. The mean HbAlc, fasting plasma glucose (FPG) and postprandial blood glucose (PPBG) levels were reduced significantly with add-on therapy $(\mathrm{P}<0.001)$. Lipid profile and creatinine were also altered significantly with the add-on therapy $(\mathrm{P}<0.001)$.

Conclusions: Add-on therapy of dapagliflozin and empagliflozin are beneficial to control the adipokines that regulate the visceral fat in overweight and obese type 2 diabetic patients. The effective therapeutic target to control adipokines with metabolic variables reduces

Manuscript submitted May 17, 2021, accepted June 15, 2021

Published online July 25, 2021

aDepartment of Biomedical Sciences, Gulf Medical University, Ajman, United Arab Emirates

bDepartment of Biochemistry, Rajah Muthiah Medical College, Annamalai University, Chidambaram, India

'Pathology Laboratory, Fakeeh University Hospital, Dubai, United Arab Emirates

dDepartment of Internal Medicine, Gulf Medical University, Ajman, United Arab Emirates

${ }^{e}$ Corresponding Author: Abid Shaheer, Department of Biomedical Sciences, P.O. Box 4184, Gulf Medical University, Ajman, United Arab Emirates. Email: abidshaheer@gmail.com

doi: https://doi.org/10.14740/jem751 body weight, obesity, cardiovascular risk and renal disease in type 2 diabetes.

Keywords: Adipokines; Visceral fat; Metformin; Dapagliflozin; Empagliflozin

\section{Introduction}

Diabetes mellitus is a metabolic disorder that occurs due to insulin deficiency or impaired insulin action, which is characterized by defective metabolisms in the body [1]. Obesity is linked with insulin resistance in peripheral tissues and is an independent risk factor for developing type 2 diabetes mellitus $[1,2]$. The risk of chronic kidney disease and cardiovascular disease has been considerably increased in type 2 diabetes with concomitant obesity. It has been noted that the development of insulin resistance may be associated with the alterations in lipid concentration in the cytoplasm of adipocytes. In addition, the alteration of adipokine secretion may lead to the development of peripheral insulin resistance or impaired function of insulin [2].

The recent evaluation by the International Diabetes Federation (IDF) estimated that the regional prevalence of diabetes mellitus in the middle east population is about $12.8 \%$, and is predicted to increase to $14.2 \%$ by 2030 [3]. The incidence of overweight in the Middle East is 48\% in males and 35\% in females and the prevalence of obesity is $24 \%$ in males and $40 \%$ in females [4]. The incidence of obesity and type 2 diabetes is a significant concern on the public health policy [5].

The body fat distribution is a major component of body weight responsible for adverse outcomes that accurately predict the prognosis and assign treatment strategies. Overweight and obesity with excess fat accumulation impair the health, leading to comorbidity occurring at different thresholds depending on the population. The estimates of body fat provide relevant information and are easily performed in clinical settings [6]. The substantial body mass index (BMI) cutoffs are associated with increased risk of body fat distribution, degree of obesity and weight. Increased risk of type 2 diabetes and premature mortality occurs well before a BMI of $30 \mathrm{~kg} / \mathrm{m}^{2}$ is reached. Therefore, the strategies to restrict weight gain will have significant health benefits in the earlier stages. In addition, the historical relationships between increased weight and comorbidity recommend better treatments for those con- 
ditions. In this regard, updated data on the health outcomes linked to overweight and obesity need to include medications and the treatment methods implemented $[6,7]$.

The alteration in metabolic pathways is closely related to the turnover of adenosine triphosphate regardless of insulin sensitivity status. The actual mechanism of systemic metabolite patterns and how the metabolisms are altered during metabolic health, adiposity and insulin sensitivity is still not well understood [8]. The adipose tissue is a highly active metabolic, endocrine organ that secretes various cytokines, collectively named adipokines. The functions of adipose tissue are enhanced by multiple proteins stimulated by metabolic alterations and enzymes involved in the steroid hormone metabolism. The adipokines are involved in altering carbohydrate or lipid metabolisms and altering insulin sensitivity through insulin signaling regulation [9].

The fatty-acid binding proteins (FABP) are intracellular lipid chaperones that help hydrophobic molecules to transport through the cell membrane. FABP4 is mainly expressed in adipose tissue and is closely associated with inflammation, insulin resistance and diabetes mellitus. FABP4 is also related to the occurrence of atherosclerosis and cardiovascular diseases [10]. The retinol-binding protein 4 (RBP4) is a protein synthesized mainly by the hepatocytes and adipocytes [11]. The RBP4 synthesized in the liver is primarily involved in the transport of retinol from the liver to peripheral tissues, and is associated with the accumulation of fat in the liver $[11,12]$. The RBP4 released in the adipose tissue is associated with the pathogenesis of insulin resistance and type 2 diabetes mellitus [11].

Visfatin is an adipokine released primarily in visceral adipose tissue, bone marrow, skeletal muscle and hepatocytes [13]. Visfatin exhibits a significant role in the physiological and pathological processes, the pathogenesis of various metabolic disorders, insulin resistance, obesity, diabetes and cardiovascular disease [13-15]. Visfatin also has an immunomodulatory function and is considered a marker of inflammation and endothelial dysfunction $[14,15]$. Adiponectin is an adipocytokine primarily secreted by the adipocytes that play a central role in protecting against insulin resistance, diabetes, obesity and cardiovascular disease [16]. The adiponectin is also produced by the liver and muscle, where it serves as an insulin-sensitizing hormone. The decreased adiponectin levels in obesity lead to low-grade inflammation, which enhances the development of metabolic disorders [17].

Metformin is an established first-line drug for the treatment of diabetes mellitus by lowering glycemia in high-risk individuals [18]. Metformin reduces hepatic glucose production, inhibits the intestinal absorption of glucose, and improves insulin sensitivity by promoting glucose uptake and utilization [18-20]. Metformin treatment is safe and effective in reducing overweight or obesity in type 2 diabetes mellitus with lifestyle interventions $[18,21]$. There is a controversy about the precise mechanism of metformin action, although many studies point out the role of the drug in reducing hepatic glucose production [22].

The sodium-glucose cotransporter 2 (SGLT2) inhibitors are a group of medicine that plays an essential role in reducing renal glucose reabsorption by preventing the action of SGLT2, thereby enhancing urinary glucose excretion [23]. SGLT2 in- hibitors are the most effective hypoglycemic medicine currently accepted as add-on therapy with metformin in subjects having diabetic complications to regulate the blood glucose level [24]. Add-on therapy of SGLT2 inhibitors with metformin is effective, safe and well-tolerated. In addition, the side effects of these combination medicines are rare compared with other hypoglycemic drugs [25].

The current study focuses mainly on the effect of add-on therapy of dapagliflozin and empagliflozin on visceral fat-associated adipokines in inadequately controlled obese and overweight type 2 diabetic patients on metformin monotherapy.

\section{Materials and Methods}

\section{Study population}

The study subjects include both males and females in the age group of $30-60$ years, newly diagnosed with type 2 diabetes mellitus in the Department of Internal Medicine, Thumbay Hospital, Ajman, United Arab Emirates. The subjects with first-line medication (metformin $1,000 \mathrm{mg}$ two times per day) were observed for a minimum of 12 weeks, and the inadequately controlled subjects were recruited for the study. The subjects who received metformin with different dosages, other hypoglycemic agents, and statins were not included. The study excluded type 1 diabetes mellitus, gestational diabetes mellitus, moderate to severe renal disease, liver disease and diabetic ketoacidosis. The subjects with normal BMI were also excluded from the study.

\section{Sample size}

A total of 60 adult type 2 diabetic patients with overweight (BMI between 25 and $29.9 \mathrm{~kg} / \mathrm{m}^{2}$ ) or obesity (BMI $\geq 30 \mathrm{~kg}$ / $\mathrm{m}^{2}$ ) were included in the study.

\section{Study settings}

Type 2 diabetic patients were identified according to the American Diabetic Association (ADA) diagnostic criteria. The data were taken before starting first-line therapy with metformin, 12 weeks after starting metformin therapy and 12 weeks after starting add-on therapy. The glycemic control was monitored by estimating hemoglobin Alc (HbAlc), fasting plasma glucose (FPG) and 2-h postprandial blood glucose (PPBG) levels. The study subjects who achieved more than $1 \%$ reduction in $\mathrm{HbA} 1 \mathrm{c}$ level or $\mathrm{HbA} 1 \mathrm{c}<7 \%$ were excluded. Information on age, lifestyle parameters, family history and treatment modalities were collected through a questionnaire after written informed consent. The height, weight, waist circumference (WC), BMI, systolic and diastolic blood pressure were recorded. A total of 181 subjects were screened for the study and 84 were uncontrolled with metformin monotherapy. Out of these 24 were withheld due to various reasons such as non-cooperation, failure in a follow-up visit, change in medi- 
cine, use of other medications for long time, surgery and infection.

\section{Duration of study}

The duration of the study was 2 years (from April 2018 to March 2020).

\section{Study instrument and validation procedure}

The ethical approval was taken from the Institutional Ethics Committee (IRB/COM/FAC/05/2018). Informed written consent was obtained from all individual participants, and the confidentiality of study subjects was maintained. This study was conducted in compliance with the ethical standards of the Gulf Medical University, Ajman, United Arab Emirates.

\section{Methodology}

The blood samples collected from the study subjects were analyzed for visfatin, adiponectin, FABP4 and RBP4 by enzyme-linked immunosorbent assay (ELISA) with the reagents supplied by BioVendor, Laboratory Medicina, Czech Republic. HbA1c level was measured with high-performance liquid chromatography (HPLC). FPG, 2-h PPBG, total cholesterol (TC), triglycerides (TG), high-density lipoprotein (HDL) cholesterol (HDL-C), low-density lipoprotein (LDL) cholesterol (LDL-C), albumin, creatinine, alanine transaminase (ALT) and aspartate transaminase (AST) were analyzed using Cobas ${ }^{\circledR}$ 6000 analyzer (Roche). BMI was calculated by dividing the weight (in kilograms) by height (in meters ${ }^{2}$ ). A sphygmomanometer was used for office blood pressure (OBP) measurement.

\section{Data analysis}

The data were expressed as the mean \pm standard deviation or frequencies. Parametric and non-parametric tests were applied to compare groups based on the type of variable and normality distribution. The z-test was applied to find the differences between the means across the groups. The effect of dapagliflozin and empagliflozin on the clinical parameters, biochemical variables and adipokines were analyzed with repeated measures analysis of covariance (ANCOVA) with treatment as the factor and change of $\mathrm{HbA1c}, \mathrm{BMI}$ and age as covariates. Data were analyzed using the Statistical Package for Social Science (SPSS Version 27.0) and a P value $<0.05$ was considered significant.

\section{Results}

Supplementary Material 1 (www.jofem.org) outlines the baseline characteristics of the study subjects. The study subjects were divided into two groups, type 2 diabetic patients with overweight and type 2 diabetic patients with obesity. The participants had an inactive lifestyle with controlled dietary habits. The mean age of participants was $45.7 \pm 7.1$ and 45.6 \pm 8.3 years in type 2 diabetes with overweight and obesity, respectively. The mean weight was $78.2 \pm 8.2 \mathrm{~kg}$, mean BMI was $27.4 \pm 1.3 \mathrm{~kg} / \mathrm{m}^{2}$ and mean $\mathrm{WC}$ was $96.3 \pm 3.7 \mathrm{~cm}$ in overweight type 2 diabetes. The mean weight was $91.1 \pm 9.0$ $\mathrm{kg}$, mean BMI was $32.5 \pm 1.3 \mathrm{~kg} / \mathrm{m}^{2}$ and mean WC was 100.6 $\pm 4.3 \mathrm{~cm}$ in obese type 2 diabetes. The mean systolic and diastolic blood pressures were $120.0 \pm 8.3$ and $76.0 \pm 6.2 \mathrm{~mm} \mathrm{Hg}$, respectively in overweight type 2 diabetes. The mean systolic and diastolic blood pressures were $122.3 \pm 8.2$ and $77.0 \pm 5.4$ $\mathrm{mm} \mathrm{Hg}$, respectively in obese type 2 diabetes.

Out of the 30 overweight participants, $10 \%$ were smokers, $43.3 \%$ had a history of hypertension and $80 \%$ had a history of hypercholesterolemia. The participants with obesity had a history of hypertension $(63.3 \%)$, hypercholesterolemia $(76.7 \%)$, and $16.7 \%$ were smokers. The subjects with overweight had a family history of diabetes mellitus $(73.3 \%)$, hypercholesterolemia $(6.7 \%)$ and hypertension $(36.7 \%)$. The subjects with obesity also had a family history of diabetes mellitus $(80 \%)$, hypercholesterolemia (6.7\%) and hypertension (46.7\%).

All the study subjects were taken metformin 1,000 mg $\left(\right.$ Glucophage ${ }^{\circledR}$ ) twice/day as first-line treatment for 12 weeks, followed by add-on therapy for 12 weeks. Among the participants, 30 subjects had taken dapagliflozin and 30 subjects had taken empagliflozin. Among the participants who received dapagliflozin, $53.33 \%$ were overweight and $46.66 \%$ were obese. Among the subjects who received empagliflozin, 46.66\% were overweight and $53.33 \%$ were obese. The subjects included 15 participants with empagliflozin/metformin $5 \mathrm{mg} / 1,000 \mathrm{mg}$ $\left(\right.$ Synjardy $\left.{ }^{\circledR}\right), 15$ with empagliflozin/metformin $12.5 \mathrm{mg} / 1,000$ $\mathrm{mg}\left(\right.$ Synjardy $\left.{ }^{\circledR}\right)$, 15 with dapagliflozin/metformin $5 \mathrm{mg} / 1,000$ $\mathrm{mg}\left(\mathrm{Xigduo}^{\circledR}\right.$ ) and 15 with dapagliflozin/metformin 10 $\mathrm{mg} / 1,000 \mathrm{mg}\left(\right.$ Xigduo $\left.^{\circledR}\right)$.

Supplementary Materials 2 and 3 (www.jofem.org) show the change from baseline to treatment with metformin, before and after add-on therapy of SGLT2 inhibitors on clinical and biochemical variables in overweight and obese type 2 diabetes patients, respectively. Add-on therapy with dapagliflozin and empagliflozin significantly reduced the mean body weight $(\mathrm{P}$ $<0.001)$ by -1.81 (95\% confidence interval (CI): $-2.13,-1.48)$, -1.83 (95\% CI: $-2.22,-1.45)$ and -1.79 (95\% CI: -2.39, -1.18) $\mathrm{kg} / \mathrm{m}^{2}$ in total overweight subjects, groups 1 and 2, respectively. A significant change is also observed in obese subjects $(\mathrm{P}<$ $0.001)$ by -2.00 (95\% CI: $-2.39,-1.61),-1.81$ (95\% CI: -2.19 , $-1.43)$ and $-2.19(95 \% \mathrm{CI}:-2.88,-1.50) \mathrm{kg} / \mathrm{m}^{2}$. Add-on therapy also exerts a beneficial reduction on the mean BMI by -0.64 (95\% CI: $-0.77,-0.52),-0.65$ (95\% CI: $-0.82,-0.49),-0.64$ (95\% CI: $-0.86,-0.41)$ in overweight participants. A similar effect is also observed in obese subjects $(\mathrm{P}<0.001)$ with a change of -0.71 (95\% CI: $-0.85,-0.58),-0.68$ (95\% CI: -0.84 , $-0.52)$ and -0.75 (95\% CI: $-0.97,-0.53)$. There was significant differences in the mean WC $(\mathrm{P}<0.001)$ by $-2.82(95 \% \mathrm{CI}$ : $-3.17,-2.48),-2.83$ (95\% CI: $-3.38,-2.27)$ and -2.82 (95\% CI: $-3.27,-2.38) \mathrm{cm}$ in overweight subjects and by $-2.91(95 \% \mathrm{CI}$ : $-3.19,-2.63),-2.87$ (95\% CI: $-3.31,-2.43)$ and -2.95 (95\% CI: $-3.37,-2.53) \mathrm{cm}$ in obesity. Add-on therapy significantly al- 
ters the mean systolic blood pressure $(\mathrm{P}<0.001)$ and diastolic blood pressure $(\mathrm{P}<0.05)$ after add-on therapy in overweight and obese type 2 diabetes. The add-on therapy with dapagliflozin was more effective $(\mathrm{P}<0.05)$ on reducing the mean systolic blood pressure compared to empagliflozin in obesity.

The mean FPG level was significantly reduced with addon therapy in the overweight study subjects $(\mathrm{P}<0.001)$ by -2.04 (95\% CI: $-2.39,-1.70),-2.20$ (95\% CI: $-2.79,-1.61),-1.89$ (95\% CI: $-2.29,-1.48) \mathrm{mmol} / \mathrm{L}$ when compared to metformin monotherapy. A similar effect was also observed in obese subjects $(\mathrm{P}<0.001)$ by -2.25 (95\% CI: $-2.57,-1.93),-2.57(95 \%$ CI: $-3.15,-1.99),-1.94$ (95\% CI: $-2.33,-1.54) \mathrm{mmol} / \mathrm{L}$. The mean PPPG level was significantly reduced $(\mathrm{P}<0.001)$ in overweight subjects by -3.29 (95\% CI: $-4.03,-2.54),-3.96$ (95\% CI: -5.37, -2.56), -2.61 (95\% CI: -3.10, -2.11) mmol/L. A similar result was also observed $(\mathrm{P}<0.001)$ in obese type 2 diabetic patients by -2.25 (95\% CI: $-2.57,-1.93),-2.57(95 \%$ CI: $-3.15,-1.99),-1.94$ (95\% CI: $-2.33,-1.54) \mathrm{mmol} / \mathrm{L}$ when compared to metformin monotherapy.

Add-on therapy exerts a beneficial reduction on $\mathrm{HbAlc}$ levels in overweight type 2 diabetic patients $(\mathrm{P}<0.001)$ by -1.14 (95\% CI: $-1.29,-1.00),-1.31$ (95\% CI: $-1.54,-1.08)$ and -0.97 (95\% CI: $-1.17,-0.77) \%$ when compared to metformin monotherapy which reduced from baseline with a reduction of -0.11 (95\% CI: $-0.19,-0.04),-0.13$ (95\% CI: $-0.25,-0.02)$ and -0.09 (95\% CI: $-0.19,0.01) \%$ within the total study subjects, group 1 and 2, respectively. The same beneficial effect was also observed in obese type 2 diabetic patients with a significant decrease $(\mathrm{P}<0.001)$ of -1.23 (95\% CI: $-1.33,-1.12)$, -1.32 (95\% CI: $-1.54,-1.11)$ and -1.13 (95\% CI: $-1.24,-1.02)$ $\%$ compared to a decrease with metformin monotherapy by -0.12 (95\% CI: $-0.18,-0.06),-0.06$ (95\% CI: $-0.15,-0.02)$, and -0.18 (95\% CI: $-0.29,-0.07) \%$.

The results also shows the alterations in the lipid profile in overweight and obese subjects with a significant decrease in mean TC $(\mathrm{P}<0.001)$, LDL-C $(\mathrm{P}<0.001)$, TG $(\mathrm{P}<0.001)$, and increase in HDL-C $(\mathrm{P}<0.001)$ after add-on therapy within the total study subjects, groups 1 and 2 . In addition, the add-on therapy also exerts a reduction $(\mathrm{P}<0.001)$ on mean creatinine level within the overweight and obese study subjects.

Supplementary Materials 4 and 5 (www.jofem.org) show the change from baseline to treatment with metformin, before and after add-on therapy of SGLT2 inhibitors on adipokines in overweight and obese type 2 diabetic patients, respectively. The mean RBP4 in overweight diabetic patients was significantly reduced $(\mathrm{P}<0.001)$ by $-5.85(95 \% \mathrm{CI}$ : $-6.87,-4.84),-6.06(95 \% \mathrm{CI}:-7.57,-4.56)$ and $-5.64(95 \%$ CI: $-7.18,-4.10) \mu \mathrm{g} / \mathrm{mL}$ compared to a non-significant $(\mathrm{P}>$ 0.05 ) change from the baseline with metformin monotherapy in total study subjects, groups 1 and 2, respectively. A similar results were observed in obese subjects with a significant decline $(\mathrm{P}<0.001)$ by $-6.16(95 \% \mathrm{CI}:-7.07,-5.26),-6.64$ (95\% CI: $-8.01,-5.27)$ and $-5.69(95 \%$ CI: $-7.03,-4.35) \mu \mathrm{g} /$ $\mathrm{mL}$ with add-on therapy in total study subjects, groups 1 and 2 , respectively. The mean RBP4 level was not significant between the study groups in overweight $(\mathrm{P}=0.439)$ and obese $(\mathrm{P}=0.463)$ subjects.

The mean FABP4 in overweight type 2 diabetic subjects was significantly reduced by -3.15 (95\% CI: $-3.65,-2.65)$,
-3.44 (95\% CI: $-4.10,-2.78)$ and -2.86 (95\% CI: $-3.70,-2.02)$ $\mathrm{ng} / \mathrm{mL}$ from the 12 th week $(\mathrm{P}<0.001)$ when compared to a non-significant $(\mathrm{P}>0.05)$ change from the baseline with metformin monotherapy in total study subjects, groups 1 and 2, respectively. The same beneficial effect was also observed in obese type 2 diabetic patients with a significant decrease $(\mathrm{P}<0.001)$ of -3.30 (95\% CI: $-3.96,-2.63),-3.32$ (95\% CI: $-4.18,-2.46)$ and -3.28 (95\% CI: $-4.36,-2.19) \mathrm{ng} / \mathrm{mL}$ with addon therapy in total study subjects, groups 1 and 2 . The mean FABP4 level was not significant between the study groups in overweight $(\mathrm{P}=0.420)$ and obese $(\mathrm{P}=0.200)$ subjects.

The mean visfatin levels in overweight subjects significantly reduced by -1.44 (95\% CI: $-1.59,-1.29),-1.38(95 \%$ CI: $-1.64,-1.12)$ and -1.51 (95\% CI: $-1.67,-1.34) \mathrm{ng} / \mathrm{mL}$ from the 12 th week $(\mathrm{P}<0.001)$ compared to a significant variation with metformin monotherapy, which is 0.13 (95\% CI: -0.06 , $0.20), 0.17$ (95\% CI: $0.06,0.28)$ and -0.10 (95\% CI: -0.01 , $-0.20) \mathrm{ng} / \mathrm{mL}$ from the baseline in total study subjects $(\mathrm{P}<$ $0.001)$, groups $1(\mathrm{P}<0.05)$ and $2(\mathrm{P}<0.05)$, respectively. A similar result was observed in obese subjects with a significant decrease by -1.95 (95\% CI: $-2.22,-1.67),-1.91(95 \%$ CI: $-2.29,-1.54)$ and -1.98 (95\% CI: $-2.41,-1.54) \mathrm{ng} / \mathrm{mL}$ with add-on therapy $(\mathrm{P}<0.001)$ in total study subjects, groups 1 and 2 , respectively. But there was no significant change in visfatin level in obese subjects with metformin monotherapy from the baseline $(\mathrm{P}>0.05)$ within the total participants, groups 1 and 2 . The mean visfatin level was not significant between the two study groups in overweight $(\mathrm{P}=0.468)$ and obese $(\mathrm{P}=0.696)$ subjects.

The mean adiponectin level in overweight subjects significantly increased by 4.28 (95\% CI: 3.90, 4.66), $4.26(95 \%$ CI: $3.67,-4.84)$ and $4.30(95 \%$ CI: $3.74,4.86) \mu \mathrm{g} / \mathrm{mL}$ from the 12 th week $(\mathrm{P}<0.001)$ in total study subjects, groups 1 and 2, respectively. But there was no significant difference in adiponectin level with metformin monotherapy from the baseline $(\mathrm{P}>0.05)$ within total study participants, groups 1 and 2 , respectively. A similar result was observed in obese subjects with a significant increase $(\mathrm{P}<0.001)$ by 5.00 (95\% CI: 4.58 , 5.42), 5.24 (95\% CI: 4.61, 5.86) and 4.77 (95\% CI: 4.12, 5.42) $\mu \mathrm{g} / \mathrm{mL}$ with add-on therapy. The mean adiponectin level was not significant between the two study groups in overweight $(\mathrm{P}$ $=0.752)$ and obese $(\mathrm{P}=0.580)$ subjects.

The adverse events observed during the study were genital infections $(\mathrm{n}=1)$ with add-on therapy of empagliflozin and urinary tract infections $(n=2)$ with add-on therapy of dapagliflozin.

\section{Discussion}

Metformin is the recommended first-line medication suggested by most guideline organizations to treat type 2 diabetes mellitus $[26,27]$. The current guidelines also recommend secondline treatment with SGLT2 inhibitors to effectively prevent the complications of type 2 diabetes mellitus [28-30]. Abdominal fat deposition induces several metabolic variations that result in increased hepatic glucose output and decreased insulin sensitivity in adipose tissue, leading to diabetes mellitus [31]. The 
current study focused on the effects of add-on therapy of dapagliflozin and empagliflozin on visceral fat-associated adipokines. The study has been conducted in both overweight and obese type 2 diabetes as the visceral fat and adiposity alter with various degrees of BMI. The participants had a routine controlled diet with an inactive lifestyle during the entire study period. The findings of the study suggest that adipokine levels are improved with the add-on therapy of dapagliflozin and empagliflozin in central obese type 2 diabetic patients insufficiently controlled with metformin.

SGLT-2 inhibitors efficiently reduce ectopic deposition of fat and body weight by promoting adipose tissue function and temporary loss of extracellular fluid accompanied by regulation of the renin-angiotensin-aldosterone system $[32,33]$. The increase in BMI is associated with high insulin resistance and low insulin sensitivity in adult type 2 diabetes mellitus [34]. WC is an index of visceral obesity approved by the World Health Organization, IDF, American Heart Association and National Institutes of Health for screening the risk of diabetes and cardiovascular disease [35]. The current study shows a beneficial effect of the add-on therapy of dapagliflozin and empagliflozin on body weight $(\mathrm{P}<0.001)$, BMI $(\mathrm{P}<0.001)$ and $\mathrm{WC}(\mathrm{P}<0.001)$ in overweight and obese type 2 diabetic patients.

The effective control of hypertension in diabetes mellitus is a target to reduce the risk of cardiovascular disease, cerebrovascular disease and renal failure. The different guidelines emphasize using various drug classes to treat hypertension in diabetic patients as the appropriate drug for hypertensive diabetics is still unclear [36]. The result of our study indicates a significant effect $(\mathrm{P}<0.05)$ of the add-on therapy on systolic and diastolic blood pressures in type 2 diabetic patients. The add-on therapy with dapagliflozin was more effective $(\mathrm{P}<$ 0.05 ) on reducing the mean systolic blood pressure compared to empagliflozin in obesity.

Previous studies reported that SGLT2 inhibitors reduce WC and visceral fat, accompanied by a decrease in leptin and an increase in adiponectin levels [37]. However, the data on changes in adipokine levels are limited, especially no data available with the add-on therapy of SGLT2 inhibitors to metformin. The RBP4 level is associated with the abnormal lipid profile and risk of cardiovascular disease, especially among subjects with obesity. The role of RBP4 in atherosclerosis has been linked with the soluble pro-inflammatory factors and the expression of pro-inflammatory cell surface adhesion molecules [38]. The current study found that serum RBP4 is significantly reduced $(\mathrm{P}<0.001)$ with add-on therapy of dapagliflozin and empagliflozin. FABP4 expressed in adipocytes and macrophages controls lipid trafficking, which is linked to inflammation and metabolic pathways. FABP4 also plays a significant role in developing atherosclerosis and insulin resistance [39]. The current study shows that FABP4 level is significantly decreased $(\mathrm{P}<0.001)$ with add-on therapy of dapagliflozin and empagliflozin indicating the effectively control of metabolic pathways.

High visceral fat in obese and overweight subjects is associated with a reduced level of adiponectin. Increased visceral fat is also related to prolonged oxidative stress and elevated inflammatory markers. Therefore, factors that lead to a reduction in visceral fat provide an essential tool for reducing obesity and related diseases [40]. The current study explains that adiponectin level is significantly elevated $(\mathrm{P}<$ 0.001 ) with add-on therapy of dapagliflozin and empagliflozin, which effectively reduces visceral fat in obese and overweight subjects.

Increased visfatin level has been observed in association with insulin resistance, type 2 diabetes mellitus and obesity. Visfatin functions as a pro-inflammatory mediator, which plays a vital role in the pathogenesis of vascular inflammation in obesity and type 2 diabetes mellitus. High visfatin levels are also related to the risk of adverse cardiac events indicating the contribution of adipokine in the inflammatory state in type 2 diabetes [41]. The current study reveals that visfatin level is significantly decreased $(\mathrm{P}<0.001)$ with add-on therapy of dapagliflozin and empagliflozin. Also, metformin monotherapy was considerably effective in reducing visfatin levels in overweight subjects $(\mathrm{P}<0.05)$, but it was not significant in obese subjects.

SGLT2 inhibitors reduce blood glucose levels by suppressing renal glucose reabsorption and enhancing urinary glucose excretion in type 2 diabetes mellitus [42]. SGLT2 inhibitors are linked with a decline in HbA1c level by $0.6-0.9 \%$ (7 - $10 \mathrm{mmol} / \mathrm{mol}$ ) regardless of background drug therapy. SGLT2 inhibitor therapy leads to a more significant reduction of HbA1c in patients with poor glycemic control due to the hyperfiltration and elevated excretion of glucose in urine [43]. Our study found that the dapagliflozin and empagliflozin are associated with a significant beneficial effect on $\mathrm{HbAlc}(\mathrm{P}<$ $0.001)$, FPG $(\mathrm{P}<0.001)$ and PPBG $(\mathrm{P}<0.001)$ levels in type 2 diabetic patients.

Dapagliflozin and empagliflozin positively reduce cardiovascular risk and heart failure in type 2 diabetic patients [44]. Recent studies highlighted the cardioprotective impact of dapagliflozin and empagliflozin in type 2 diabetic subjects with authenticated cardiovascular disease [44-46]. Our study found that the add-on therapy has a significant beneficial effect $(\mathrm{P}$ $<0.001$ ) on lipid profile in type 2 diabetic patients agreeing to the findings of studies exploring the role of SGLT2 inhibitors in preventing cardiovascular complications. Overall, there were no significant differences in the results between the dapagliflozin and empagliflozin groups due to the same mechanism of action of SGLT2 inhibitors. The study provides new insight to target visceral fat-associated adipokines in central obese subjects to control the risk factors and to prevent the complications of type 2 diabetes mellitus.

There were few limitations to this study. The sample size was less because we have selected the participants who have taken only metformin therapy. The study also excluded the participants who changed the medicine during the study period. Also, participants' lifestyle was different due to the inclusion of different nationalities with various dietary habits. The participants who developed adverse clinical events during the study period were excluded from participating in the study.

\section{Conclusions}

The adipokine levels are improved with the add-on therapy of 
dapagliflozin and empagliflozin in central obese type 2 diabetic patients insufficiently controlled with metformin. Both dapagliflozin and empagliflozin are beneficial in controlling adipokines and regulating the visceral fat in overweight and obese type 2 diabetic patients. Add-on therapy of dapagliflozin and empagliflozin effectively reduce the visceral fat in overweight and obese subjects. The effective therapeutic target to control visceral fat-associated adipokines with metabolic variables reduces body weight, obesity, cardiovascular risk and renal disease in type 2 diabetes.

\section{Supplementary Material}

Suppl 1. Baseline characteristics of the study subjects.

Suppl 2. Change from baseline to treatment with metformin, before and after add-on therapy of SGLT2 inhibitors on clinical and biochemical variables in overweight type 2 diabetes.

Suppl 3. Change from baseline to treatment with metformin, before and after add-on therapy of SGLT2 inhibitors on clinical and biochemical variables in obese type 2 diabetes.

Suppl 4. Change from baseline to treatment with metformin, before and after add-on therapy of SGLT2 inhibitors on adipokines in overweight type 2 diabetes.

Suppl 5. Change from baseline to treatment with metformin, before and after add-on therapy of SGLT2 inhibitors on adipokines in obese type 2 diabetes.

\section{Acknowledgments}

We acknowledge the laboratory staff and biomedical engineer in Thumbay Laboratory, Gulf Medical University for their help and support during the research work.

\section{Financial Disclosure}

There was no specific funding source to be mentioned.

\section{Conflict of Interest}

The authors report no conflict of interest.

\section{Informed Consent}

All subjects provided written informed consent.

\section{Author Contributions}

AS has done the research work and the manuscript writing. AK has supervised the complete study and involved in the manuscript editing. PM also guided the research and provided facil- ity support for the study. MJ and SB are the clinicians whose patients were enrolled in the study.

\section{Data Availability}

The authors declare that data supporting the findings of this study are available within the article.

\section{References}

1. Khemka VK. Metabolic risk factors in obesity and diabetes mellitus: implications in the pathogenesis and therapy. Integr Obesity Diabetes. 2017:3(3):1-4.

2. Kocot J, Dziemidok P, Kielczykowska M, Hordyjewska A, Szczesniak G, Musik I. Adipokine profile in patients with type 2 diabetes depends on degree of obesity. Med Sci Monit. 2017;23:4995-5004.

3. Karuranga S, Malanda B, Saeedi P, Salpea P (Eds.). The IDF Diabetes Atlas, 9th edition. Int Diab Feder. 2019; p. 62-77.

4. Sulaiman N, Elbadawi S, Hussein A, Abusnana S, Madani A, Mairghani M, Alawadi F, et al. Prevalence of overweight and obesity in United Arab Emirates Expatriates: the UAE National Diabetes and Lifestyle Study. Diabetol Metab Syndr. 2017;9:88.

5. de Oliveira CM, Pavani J, Krieger JE, de Oliveira Alvim R, Mourao-Junior CA, da Costa Pereira A. Body adiposity index in assessing the risk of type 2 diabetes mellitus development: the Baependi Heart Study. Diabetol Metab Syndr. 2019;11:76.

6. Purnell JQ. Definitions, classification, and epidemiology of obesity. In: Feingold KR, Anawalt B, Boyce A, Chrousos G, de Herder WW, Dhatariya K, et al., eds. Endotext. South Dartmouth (MA); 2000.

7. Weir CB, Jan A. BMI classification percentile and cut off points. In: StatPearls. Treasure Island (FL); 2021.

8. Grapov D, Fiehn O, Campbell C, Chandler CJ, Burnett DJ, Souza EC, Casazza GA, et al. Exercise plasma metabolomics and xenometabolomics in obese, sedentary, insulin-resistant women: impact of a fitness and weight loss intervention. Am J Physiol Endocrinol Metab. 2019;317(6):E999-E1014.

9. Shi J, Fan J, Su Q, Yang Z. Cytokines and abnormal glucose and lipid metabolism. Front Endocrinol (Lausanne). 2019;10:703.

10. von Jeinsen B, Ritzen L, Vietheer J, Unbehaun C, Weferling $\mathrm{M}$, Liebetrau $\mathrm{C}$, Hamm $\mathrm{CW}$, et al. The adipokine fatty-acid binding protein 4 and cardiac remodeling. Cardiovasc Diabetol. 2020;19(1):117.

11. Chen X, Shen T, Li Q, Chen X, Li Y, Li D, Chen G, et al. Retinol Binding Protein-4 Levels and Non-alcoholic Fatty Liver Disease: A community-based cross-sectional study. Sci Rep. 2017;7:45100.

12. Fan J, Yin S, Lin D, Liu Y, Chen N, Bai X, Ke Q, et al. Association of Serum Retinol-Binding Protein 4 Levels and the Risk of Incident Type 2 Diabetes in Subjects With 
Prediabetes. Diabetes Care. 2019;42(8):1574-1581.

13. Heo YJ, Choi SE, Jeon JY, Han SJ, Kim DJ, Kang Y, Lee $\mathrm{KW}$, et al. Visfatin induces inflammation and insulin resistance via the NF-kappaB and STAT3 signaling pathways in hepatocytes. J Diabetes Res. 2019;2019:4021623.

14. Zhang Y, Huo Y, He W, Liu S, Li H, Li L. Visfatin is regulated by interleukin6 and affected by the PPARgamma pathway in BeWo cells. Mol Med Rep. 2019;19(1):400406.

15. Zheng LY, Xu X, Wan RH, Xia S, Lu J, Huang Q. Association between serum visfatin levels and atherosclerotic plaque in patients with type 2 diabetes. Diabetol Metab Syndr. 2019;11:60.

16. Achari AE, Jain SK. Adiponectin, a therapeutic target for obesity, diabetes, and endothelial dysfunction. Int J Mol Sci. 2017;18(6):1321.

17. Forny-Germano L, De Felice FG, Vieira M. The Role of leptin and adiponectin in obesity-associated cognitive decline and Alzheimer's disease. Front Neurosci. 2018;12:1027.

18. Aroda VR, Ratner RE. Metformin and type 2 diabetes prevention. Diabetes Spectr. 2018;31(4):336-342.

19. Rena G, Hardie DG, Pearson ER. The mechanisms of action of metformin. Diabetologia. 2017;60(9):1577-1585.

20. LaMoia TE, Shulman GI. Cellular and molecular mechanisms of metformin action. Endocr Rev. 2021;42(1):7796.

21. Pu R, Shi D, Gan T, Ren X, Ba Y, Huo Y, Bai Y, et al. Effects of metformin in obesity treatment in different populations: a meta-analysis. Ther Adv Endocrinol Metab. 2020;11:2042018820926000.

22. Sanchez-Rangel E, Inzucchi SE. Metformin: clinical use in type 2 diabetes. Diabetologia. 2017;60(9):1586-1593.

23. Simes BC, MacGregor GG. Sodium-Glucose Cotransporter-2 (SGLT2) Inhibitors: A Clinician's Guide. Diabetes Metab Syndr Obes. 2019;12:2125-2136.

24. Kurinami N, Sugiyama S, Nishimura H, Morita A, Yoshida A, Hieshima K, Miyamoto F, et al. Clinical factors associated with initial decrease in body-fat percentage induced by add-on sodium-glucose co-transporter 2 inhibitors in patient with type 2 diabetes mellitus. Clin Drug Investig. 2018;38(1):19-27.

25. Muller-Wieland D, Kellerer M, Cypryk K, Skripova D, Rohwedder K, Johnsson E, Garcia-Sanchez R, et al. Efficacy and safety of dapagliflozin or dapagliflozin plus saxagliptin versus glimepiride as add-on to metformin in patients with type 2 diabetes. Diabetes Obes Metab. 2018;20(11):2598-2607.

26. Flory J, Lipska K. Metformin in 2019. JAMA. 2019;321(19):1926-1927.

27. Baker C, Retzik-Stahr C, Singh V, Plomondon R, Anderson V, Rasouli N. Should metformin remain the first-line therapy for treatment of type 2 diabetes? Ther Adv Endocrinol Metab. 2021;12:2042018820980225.

28. Thein D, Christiansen MN, Mogensen UM, Bundgaard JS, Rorth R, Madelaire C, Fosbol EL, et al. Add-on therapy in metformin-treated patients with type 2 diabetes at moderate cardiovascular risk: a nationwide study. Cardiovasc Diabetol. 2020;19(1):107.
29. Mosenzon O, Pollack R, Raz I. Treatment of type 2 diabetes: from "Guidelines" to "Position Statements" and back: recommendations of the Israel National Diabetes Council. Diabetes Care. 2016;39(Suppl 2):S146-153.

30. Kalra S, Kesavadev J, Chadha M, Kumar GV. Sodiumglucose Cotransporter-2 Inhibitors in Combination with Other Glucose-lowering Agents for the Treatment of Type 2 Diabetes Mellitus. Indian J Endocrinol Metab. 2018;22(6):827-836.

31. Frankenberg ADV, Reis AF, Gerchman F. Relationships between adiponectin levels, the metabolic syndrome, and type 2 diabetes: a literature review. Arch Endocrinol Metab. 2017;61(6):614-622.

32. Sheu WHH, Chan SP, Matawaran BJ, Deerochanawong C, Mithal A, Chan J, Suastika K, et al. Use of SGLT-2 inhibitors in patients with type 2 diabetes mellitus and abdominal obesity: an Asian perspective and expert recommendations. Diabetes Metab J. 2020;44(1):11-32.

33. Schork A, Saynisch J, Vosseler A, Jaghutriz BA, Heyne N, Peter A, Haring HU, et al. Effect of SGLT2 inhibitors on body composition, fluid status and renin-angiotensinaldosterone system in type 2 diabetes: a prospective study using bioimpedance spectroscopy. Cardiovasc Diabetol. 2019;18(1):46.

34. Zhao Q, Laukkanen JA, Li Q, Li G. Body mass index is associated with type 2 diabetes mellitus in Chinese elderly. Clin Interv Aging. 2017;12:745-752.

35. Fang H, Berg E, Cheng X, Shen W. How to best assess abdominal obesity. Curr Opin Clin Nutr Metab Care. 2018;21(5):360-365.

36. Grossman A, Grossman E. Blood pressure control in type 2 diabetic patients. Cardiovasc Diabetol. 2017;16(1):3.

37. Garvey WT, Van Gaal L, Leiter LA, Vijapurkar U, List J, Cuddihy R, Ren J, et al. Effects of canagliflozin versus glimepiride on adipokines and inflammatory biomarkers in type 2 diabetes. Metabolism. 2018;85:32-37.

38. Anna MR, Marzena SZ, Aleksandra Z, Piotr E, Eliana BS, Agnieszka Z, et al. Is the retinol-binding protein 4 a possible risk factor for cardiovascular diseases in obesity? Int J Mol Sci. 2020;21(15):5229.

39. Furuhashi M. Fatty acid-binding protein 4 in cardiovascular and metabolic diseases. J Atheroscler Thromb. 2019;26(3):216-232.

40. Gariballa S, Alkaabi J, Yasin J, Al Essa A. Total adiponectin in overweight and obese subjects and its response to visceral fat loss. BMC Endocr Disord. 2019;19(1):55.

41. Landecho MF, Tuero C, Valenti V, Bilbao I, de la Higuera M, Fruhbeck G. Relevance of leptin and other adipokines in obesity-associated cardiovascular risk. Nutrients. 2019;11(11):2664.

42. Chen MB, Wang H, Cui WY, Xu HL, Zheng QH. Effect of SGLT inhibitors on weight and lipid metabolism at 24 weeks of treatment in patients with diabetes mellitus: A systematic review and network meta-analysis. Medicine (Baltimore). 2021;100(6):e24593.

43. Thomas MC, Cherney DZI. The actions of SGLT2 inhibitors on metabolism, renal function and blood pressure. Diabetologia. 2018;61(10):2098-2107.

44. Kale S, Tahrani AA. Sodium-glucose co-transporter-2 in- 
hibitors in patients with type 2 diabetes mellitus without established cardiovascular disease: Do they have a role in primary prevention? Metabol Open. 2021;10:100082.

45. Rehman SU, Rahman F. Evidence-based clinical review on cardiovascular benefits of SGLT2 (Sodium-Glucose
Co-Transporter Type 2) inhibitors in type 2 diabetes mellitus. Cureus. 2020;12(8):e9655.

46. Rabizadeh S, Nakhjavani M, Esteghamati A. Cardiovascular and renal benefits of SGLT2 inhibitors: a narrative review. Int J Endocrinol Metab. 2019;17(2):e84353. 